\title{
CXCR4/CXCR7 gene-silencing suppresses ovarian cancer cell proliferation and tumour growth through modulation of the CXCL12-CXCR4/CXCR7 chemokine axis and activation of MAPK signalling pathway
}

Fan Yang ${ }^{1,2}$, Li Zhang ${ }^{1,2}$, Xian-Gen Xia ${ }^{1,2}$, Li Ma ${ }^{1,2}$, Sheng-Jun Cheng ${ }^{1,2}$, Xing-Li ji ${ }^{1,2}$

\begin{abstract}
${ }^{1}$ Department of Pathology, Chengdu Fifth People's Hospital, Chengdu, Sichuan, China 2Department of Pathology, The Fifth Affiliated People's Hospital of Chengdu University of Traditional Chinese Medicine, Chengdu, Sichuan, China
\end{abstract}

Submitted: 29 April 2020

Accepted: 18 August 2020

Arch Med Sci

DOI: https://doi.org/10.5114/aoms.2020.100657

Copyright @ 2020 Termedia \& Banach

\begin{abstract}
Introduction: Ovarian cancer is the most common malignant cancer among females. This study aimed to investigate role of the CXCL12-CXCR4/CXCR7 chemokine axis in ovarian cancer.

Material and methods: SKOV3 cells were transfected with siCXCR4/siCXCR7 plasmids and divided into SKOV3, LV3, LV3-siCXCR4, and LV3-siCXCR7 groups. Cell viability and apoptosis were examined using MTT and flow cytometry. ELISA was conducted to evaluate vascular-endothelial growth factor-A (VEGF-A), interleukin-6 (IL-6), and interleukin-8 (IL-8) levels. SKOV3 cells were transplanted into mice to establish a Xenograft model. The tumour volume of the Xenograft model was recorded. CD31, matrix-metalloprotein-9 (MMP-9), vimentin, and E-cadherin expressions in SKOV3 cells and tumour tissues were evaluated with quantitative real-time PCR (qRT-PCR) and western blot. Immunohistochemistry assay was used to detect VEGF-A, CD31, MMP-9, vimentin, and E-cadherin expression.

Results: SKOV3 cells strongly expressed CXCR4 and CXCR7. CXCL12 significantly enhanced cell viability, and CXCR4/CXCR7 inhibitor remarkably reduced cell viability of SKOV3 $(p<0.05)$. CXCR4/CXCR7 gene-silencing significantly decreased SKOV3 viability compared to the SKOV3 or LV3 group $(p<0.05)$. CXCR4/CXCR7 gene-silencing modulated cell cycle and increased apoptosis rates compared to SKOV3 or LV3 group $(p<0.05)$. CXCR4/CXCR7 gene-silencing inhibited VEGF-1, IL-6, and IL-8 secretion. CXCR4/CXCR7 gene-silencing modulated levels of CD31, MMP-9, vimentin, and E-cadherin. CXCR4/CXCR7 gene-silencing suppressed $p$-ERK1/2 $(p<0.05)$ and strengthened inhibitive effects of CXCR4/CXCR7 inhibitor on p-ERK1/2. CXCR4/CXCR7 gene-silencing reduced tumour volume and regulated tumourigenesis-associated molecules in the mouse Xenograft model.

Conclusions: CXCR4/CXCR7 gene-silencing could suppress ovarian cancer cell proliferation and tumour growth through modulation of the CXCL12CXCR4/CXCR7 chemokine axis and mitogen-activated protein kinase (MAPK) signalling pathway.
\end{abstract}

Key words: CXCL12-CXCR4/CXCR7 chemokine axis, ovarian cancer, MAPK signalling pathway, gene-silencing, tumour growth.
Corresponding author:

Prof. Fan Yang

Department of Pathology

Chengdu Fifth

People's Hospital

Chengdu, Sichuan, China

E-mail: yangfan03@126.com 


\section{Introduction}

Ovarian cancer is one of the most frequently occurring malignant cancers and the fifth leading reason for cancer-associated death among females in the world [1-3]. About 1 in 54 women have ovarian cancer in their lifetime [4]. The 5-year survival rate for stage III and IV ovarian cancer is only $30 \%$, and for stage I and II it is up to $90 \%$ [5]. However, when ovarian cancer is diagnosed, more then $70 \%$ patients have progressed to the advanced stages (stage III or IV) [6]. Therefore, it is crucial to promote the clinical outcomes of ovarian cancer by developing novel therapeutic approaches to guide the clinical treatment.

The CXCL12-CXCR4/CXCR7 chemokine axis has been proven to play an integral role in tumour development and progression [7] and is associated with epithelial-mesenchymal transition (EMT) in many cancers $[8,9]$. Stromal cell-derived factor 1 (CXCL12), a kind of cytokine, belongs to the CXCchemokine family and plays a critical role in tumour cell proliferation, tumour growth, tumour angiogenesis, and tumour distant metastases in many malignant cancers, such as prostate cancer [10], glioma [11], breast cancer [12], and lung cancer [12]. CXCL12 is a promising chemo-attractant for haematopoietic cells and could modulate tumour growth-associated signalling pathways by binding two receptors (CXCR4 and CXCR7 molecule) $[13,14]$. In fact, both the CXCR4 and CXCR7 molecules have been proven to demonstrate trigger cancer progression and induce metastasis. Therefore, taken together, evidence shows that the CXCL12-CXCR4/CXCR7 chemokine axis is composed and involved in tumourigenesis, development, and progression [15]. Meanwhile, the binding of CXCL12 to CXCR4 or CXCR7 could also activate the ERK signalling pathway, which contributes to the tumour cell invasion, migration, and tumour growth [16]. However, there are limited studies exploring the effects of the CXCL12-CXCR4/CXCR7 chemokine axis in the progression of ovarian cancer. We believe that the CXCL12-CXCR4/CXCR7 chemokine axis could serve as a promising prognostic and targeting biomarker for ovarian cancer.

In the present study, we administrated CXCL12 and silenced the CXCR4/CXCR7 gene in ovarian cancer cell line SKOV3, and explored the functions of CXCL12-CXCR4/CXCR7 chemokine axis in ovarian cancer.

\section{Material and methods}

\section{Cell culture}

The human ovarian cancer cell line SKOV3 was purchased from the Cell Bank of Type Culture Collection (CAS, Shanghai, China). The SKOV3 cells were cultured in Roswell Park Memorial Institute
1640 (RPMI-1640, Gibco BRL. Co. Ltd., Grand Island, New York, USA) containing 10\% foetal bovine serum (FBS, Gibco BRL. Co. Ltd.) and 1\% penicillin/ streptomycin (Beyotime Biotech, Shanghai, China) in a humidified atmosphere with $\mathrm{CO}_{2}$ at $37^{\circ} \mathrm{C}$.

\section{Small interfering plasmid construction, lentivirus packaging, and transfection}

The oligonucleotide sequences for the small interfering CXCR4 (siCXCR4, three candidates), small interfering (siCXCR7, three candidates), and small interfering control (shNC) are illustrated in Figure $1 \mathrm{~A}$. The efficacy for silencing CXCR4 or CXCR7 gene in SKOV3 cells was demonstrated in Figure $1 \mathrm{~B}$. The pre-experiments showed that the siCXCR4-Homo-701 was the optimal sequence for silencing the CXCR4 gene and that siCXCR7Homo-1199 was the optimal sequence for silencing the CXCR7 gene. Also, the gene sequencing illustrated that the sequences for all siCXCR4, SiCXCR57, and shNC were correct (Figure $1 \mathrm{C}$ ). All of the above oligonucleotides were synthesised by GenePhama Co. Ltd. (Shanghai, China).

pG-LV3 lentiviral vector (LV3), purchased from GenePhama Co. Ltd. (Shanghai, China), was used to generate LV3-siCXCR4 and LV3-siCXCR7 plasmid. Briefly, the LV3-siCXCR4 and LV3-siCXCR7 plasmid-associated DNA double-chain was artificially synthesised using the above oligonucleotides as template sequences. Subsequently, the synthesised double-chain sequences were subcloned into PG-LV3 plasmid to generate LV3-siCXCR4 and LV3-siCXCR7 plasmid.

Finally, SKOV3 cells were packaged by employing 293T cells according to the method elsewhere. In brief, about $24 \mathrm{~h}$ post viral plasmid transfection, 293T cells were seeded into a 10-cm dish. The LV3siCXCR4, LV3-siCXCR7 plasmid, and packing plasmids (containing elements of PG-p1-VSVG, PG-P2REV, PG-P3-RRE) were transfected with RNAi-mate Kit (GenePhama Co., Ltd., Shanghai, China), as instructed by the manufacturer. The processes for transfection were conducted according to a previous study [17], with a few modifications. Finally, the efficacy of LV3-siCXCR4 and LV3-siCXCR7 lentivirus transfection was determined using immunofluorescence assay.

\section{MTT assay}

The SKOV3 cells were cultured and seeded into 96-well plates (Corning-Costar, Corning, NY, USA) at a density of $5 \times 10^{3}$ cells per well. Then, the cells were incubated with the above plasmids or regents for 24 h, 48 h, and 72 h. Cell viability was evaluated using 3-[4,5-dimethylthiazol-2yl]-2,5-diphenyl tetrazolium bromide assay (MTT, Sigma, St. Louis, MO, USA), according to a previ- 
A

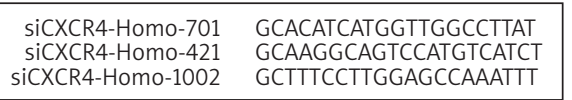

ShNC 5'-TTCTCCGAACGTGTCACGT-3'

B

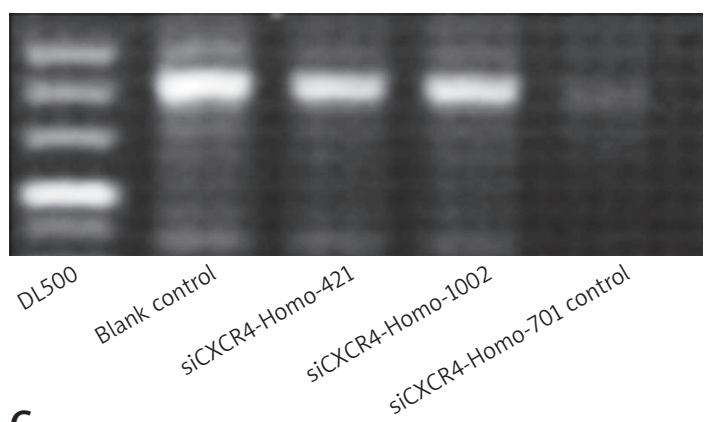

C

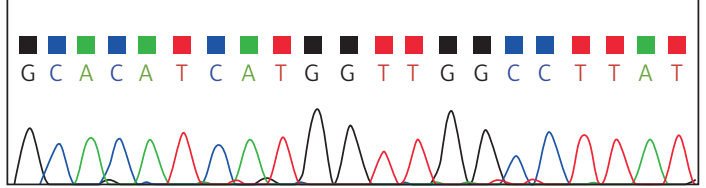

LV3-siCXCR4-Homo-701

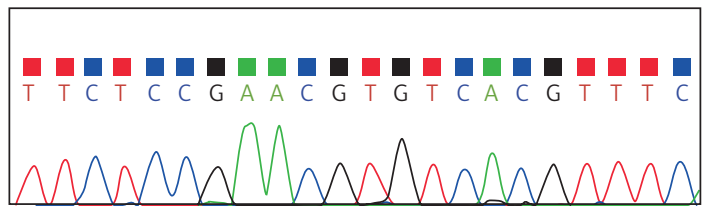

LV3-shNC
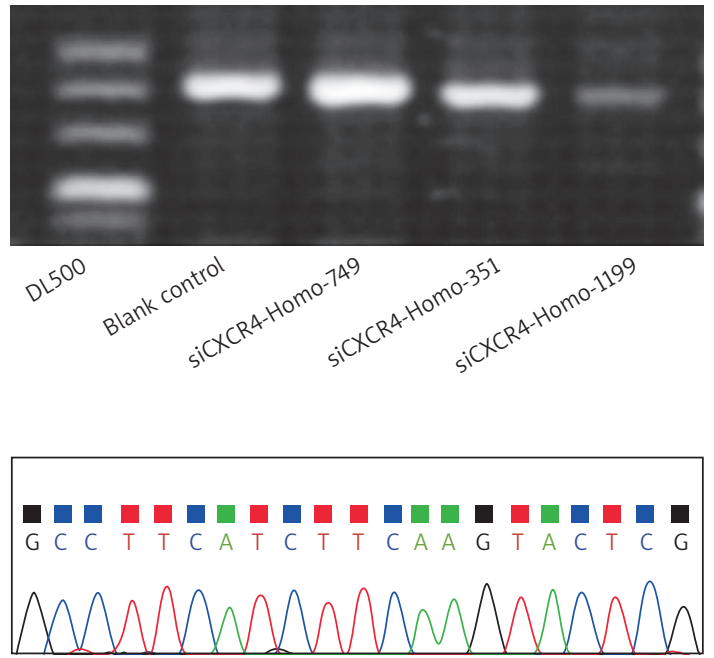

LV3-siCXCR7-Homo-1199

Figure 1. Synthesis and identification of the CXCR4/ CXCR7 small interfering RAN. A - Oligonucleotide sequences for small interfering CXCR4/CXCR7 (siCXCR4/siCXCR7). B - Screening for the optimal silencing sequence of siCXCR4/siCXCR7 using agarose gel electrophoresis. C - Gene sequencing for the synthesised siCXCR4/siCXCR7 ous study [18]. Briefly, the cells were treated with MTT for $4 \mathrm{~h}$ at $37^{\circ} \mathrm{C}$. Then, the culture medium was discarded, and MTT formazan crystals were dissolved into $150 \mu \mathrm{l}$ dimethyl sulfoxide (DMSO, Amresco Inc., Solon, OH, USA) for $10 \mathrm{~min}$. The absorbance was measured using a microplate reader (Mode: MK3, Thermo Fisher Scientific, Waltham, MA, USA) at a wavelength of $490 \mathrm{~nm}$. All of the MTT procedures were repeated at least six times.

\section{Cell cycle analysis}

The SKOV3 cells were incubated with the above plasmids or regents and fixed using $80 \%$ cold-ethanol and treated with $0.5 \%$ Triton X-100 (supplementing with $1 \mathrm{mg} / \mathrm{ml}$ RNase A) for $30 \mathrm{~min}$ at $37^{\circ} \mathrm{C}$. The cell cycle of SKOV3 cells was evaluated using flow cytometry with propidium iodide $(\mathrm{Pl})$ staining as reported in a previously published study [19].

\section{Flow cytometry analysis for apoptosis}

In this study, the apoptosis of SKOV3 cells was examined using flow cytometry assay and commercial Annexin V-PE/7-ADD Apoptosis Detection Kit (Cat. No. BD 559763, BD biosciences, Franklin Lakes, NJ, USA) as instructed by the manufacturer.
Briefly, SKOV3 cells were harvested and washed with PBS (Beyotime Biotech, Shanghai, China) and then suspended at final density of $5 \times 10^{3}$ cells/ $\mathrm{ml}$ RPMI-1640. A total of $100 \mu \mathrm{l}$ of cell suspension was added into the 5-ml tube and incubated with $5 \mu \mathrm{l}$ Annexin V-PE and $1 \mu \mathrm{l}$ 7-ADD in the dark for 30 min. Finally, the prepared SKOV3 cells were analysed using a FACSCalibur flow cytometer (BD biosciences, Franklin Lakes, NJ, USA).

\section{Enzyme-link immunosorbent assay (ELISA)}

The vascular endothelial growth A (VEGF-A), interleukin 6 (IL6), and interleukin 8 (IL-8) in culture medium of SKOV 3 cells were examined using VEGF-A ELISA kit (Cat. No. DVE00), IL-6 ELISA Kit (Cat. No. D6050), and IL-8 ELISA Kit (Cat. No. D8000C), according to the manufacturers' instructions. All of the above three ELISA kits were purchased from BD biosciences (Franklin Lakes, NJ, USA).

\section{Mouse Xenograft model establishment}

The mouse Xenograft model for ovarian cancer was established using the SKOV3 cells. In brief, BALB/C mice (Beijing Huafukang Biotech Co. Ltd., Beijing, China), aging from 4 to 8 weeks, weight- 
ing from 15 to $20 \mathrm{~g}$, were randomly divided into three groups: SKOV3 group $(n=4$, without any treatment), LV3 group ( $n=4$, administrated with SKOV3 cells with blank LV3 plasmid), LV3-siCXCR4 group (administrated with SKOV3 cells containing siCXCR4 plasmid), and LV3-siCXCR7 group ( $n=4$, administrated with SKOV3 cells containing siCXCR7 plasmid). All mice were housed in specificpathogen-free (SPF) conditions. For mice in every group, SKOV3 cells $\left(1 \times 10^{7}\right.$ cells $)$ were injected into the right flank. The mice were monitored and the tumour length and width were recorded every 2 days. The tumour volume was calculated as the following formula: tumour volume $\left(\mathrm{mm}^{3}\right)=$ tumour length $(\mathrm{mm}) \times$ tumour width $\left(\mathrm{mm}^{2}\right) / 2$. After the experiments, the mice were euthanised and the tumour tissues were removed for immunohistochemistry assay.

All of the protocols for the animal experiments were approved by the Ethics Committee of the Chengdu Fifth People's Hospital, Chengdu, China.

\section{Quantitative real-time PCR assay (qRT-PCR)}

Total RNAs in both SKOV3 cells and tumour tissues were extracted using Trizol kits (Beyotime Biotech Co. Ltd., Shanghai, China). The complementary DNAs (cDNAs) were also synthesised using SuperScript III CellsDirect cDNA Synthesis Kit (Cat. No. 18080200, Thermo Scientific Pierce, Rockford, IL, USA) according to the manufacturer's instruction. The mRNA transcriptions of CD31, matrix metalloprotein 9 (MMP-9), vimentin, and E-cadherin were determined using SYBR Green I PCR kit (Western Biotech., Chongqing, China) using specific primers (Table I). Amplified products for the above genes were analysed with a GDS8000 gel scanning system (UVP, Sacramento, CA, USA) according to the manufacturer's instructions. Final gene expressions were calculated and analysed with the $2^{-\Delta \Delta c t}$ method, as described in a previous study [20].

\section{Western blot assay}

The SKOV3 cells and tumour tissues were lysed using Cell Lysis Buffer for Western (Cat. No. P0013, Beyotime Biotech, Shanghai, China). The protein lysates for each group (20 $\mu \mathrm{g}$ per sample) were separated using 12\% SDS-PAGE (Sangon Biotech Co. Ltd., Shanghai, China). Then, protein in gels were electro-transferred onto PVDF membranes (Bio-Rad Laboratories, Hercules, CA, USA) with Trans-Blot SD Semi-Dry Electrophoretic Transfer (Mode: 170-3940, Bio-Rad Laboratories). PVDF membranes were then incubated using rabbit anti-CXCR monoclonal antibody $(1: 3000$, Cat. No. ab181020), rabbit anti-CXCR7 polyclonal antibody (1: 2000; Cat. No. ab117836), rabbit anti-CD31 polyclonal antibody (Cat. No. ab28364), rabbit anti-MMP-9 polyclonal antibody (Cat. No. ab38898), rabbit anti-vimentin monoclonal antibody (Cat. No. ab922547), rabbit anti-E-cadherin monoclonal antibody (Cat. No. ab76319), rabbit anti-ERK1/2 polyclonal antibody (Cat. No. ab212206), rabbit anti-p-ERK1/2 polyclonal antibody (Cat. No. ab214362), and rabbit anti-human GAPDH monoclonal antibody (1 : 3000; Cat. No. ab181602) at $4^{\circ} \mathrm{C}$ overnight. All of the above first antibodies were obtained from Abcam Biotech (Cambridge, Massachusetts, USA). Then, the PVDF membranes were continuously incubated using horse radish peroxidase (HRP)-labelled goat anti-rabbit IgG ( $1: 2000$, Cat. No. AQ132P, Sigma-Aldrich, St. Louis, Missouri, USA) at room temperature for $2 \mathrm{~h}$. Eventually, the PVDF membranes were treated with a Pierce ECL Kit (Cat. No. 32106, Thermo Scientific Pierce, Rockford, IL, USA) for 2 min in the dark at

Table I. Primers for the qRT-PCR assay

\begin{tabular}{|c|c|c|c|}
\hline Genes & & Sequences & Length $[\mathrm{bp}]$ \\
\hline \multirow[t]{2}{*}{ CD31 } & Forward & 5'-CACCAAGATAGCCTCAAAGTCG-3' & 116 \\
\hline & Reverse & 5'-GCTGGGAGAGCATTTCCACATAC-3' & \\
\hline \multirow[t]{2}{*}{ MMP-9 } & Forward & 5'-CGAACTTTGACAGCGACAAGA-3' & 214 \\
\hline & Reverse & 5'-TCAGGGCGAGGACCATAGAG-3' & \\
\hline \multirow[t]{2}{*}{ Vimentin } & Forward & 5'-AAATGGCTCGTCACCTTCG-3' & 161 \\
\hline & Reverse & 5'-CAGATTAGTTTCCCTCAGGTTCA-3' & \\
\hline \multirow[t]{2}{*}{ E-cadherin } & Forward & 5'-GCCCCGCCTTATGATTCTC-3' & 130 \\
\hline & Reverse & 5'-TGCCCCATTCGTTCAAGTAG-3' & \\
\hline \multirow[t]{2}{*}{$\beta$-actin } & Forward & 5'-TGACGTGGACATCCGCAAAG-3' & 205 \\
\hline & Reverse & 5'-CTGGAAGGTGGACAGCGAGG-3' & \\
\hline
\end{tabular}


room temperature. The western blotting band images were analysed using the LabworksTM Analysis Software (version: 4.0, Labworks, Upland, CA, USA).

\section{Immunohistochemistry assay}

The tumour tissues were extracted and fixed using 4\% paraformaldehyde (Sangon Biotech) for $20 \mathrm{~min}$ at room temperature. Then, the tumour tissues were sliced into sections with thickness of $5 \mu \mathrm{m}$, and $3 \%$ hydrogen peroxide was used to inactivate the endogenous peroxidase for $10 \mathrm{~min}$. The tumour tissue sections were then blocked using 5\% goat serum albumin (BSA, Hyclone, Logan, UT, USA) for $20 \mathrm{~min}$ at room temperature. The tumour tissue sections were also incubated with rabbit anti-CD31 polyclonal antibody (Cat. No. ab28364), rabbit anti-MMP-9 polyclonal antibody (Cat. No. ab38898), rabbit anti-vimentin monoclonal antibody (Cat. No. ab922547), and rabbit anti-E-cadherin monoclonal antibody (Cat. No. ab76319) at $4^{\circ} \mathrm{C}$ overnight. Subsequently, the sections were treated using Biotin-conjugated goat anti-rabbit IgG (1 : 1000, Cat. No. ab6702, Abcam Biotech) for $1 \mathrm{~h}$ at room temperature. Eventually, the sections were immersed in alkaline phosphatase-labelled streptavidin (ZSGB Bio. Inc. Co., Beijing, China). The images of sections were captured with an inverted fluorescence microscope (Model: CKX31; Olympus, Toyoko, Japan).

A

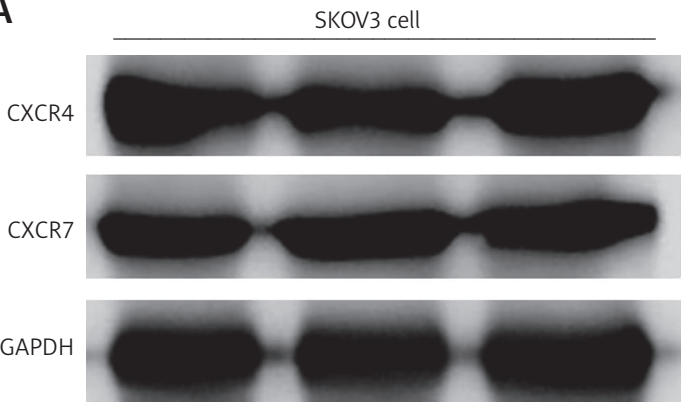

B

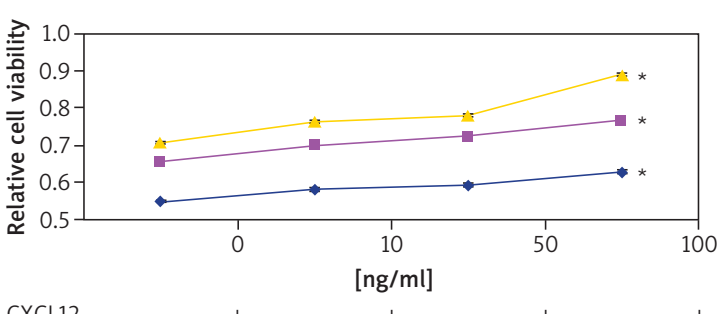

CXCL12 $-24 h-48 h-42 h$

\section{Statistical analysis}

In this study, the data was assigned as mean \pm standard deviation (SD) and analysed with professional SPSS software (version: 19.0, SPSS Inc., Chicago, IL, USA). ANOVA validated by Tukey's posthoc test was employed to compare differences among multiple groups, and Student's t-test was used to compare differences between two groups. All experiments or tests were repeated at least six times. The $p$-value less than 0.05 was assigned as statistical difference.

\section{Results}

\section{SKOV3 cells highly expressed CXCR4 and CXCR7 molecules}

In order to determine the endogenous expression of CXCR4 and CXCR7 molecules in the SKOV3 cells, western blot assay was conducted in this study. The results indicated that SKOV3 cells highly expressed CXCR4 and CXCR7 (Figure $2 \mathrm{~A}$ ), which suggests that both CXCR4 and CXCR7 are resident proteins in SKOV3 cells.

CXCL12 administration enhanced cell viability and CXCR4/CXCR7 inhibitor reduced cell viability of SKOV3 cells

The results showed that the CXCL12 administrations (10 ng/ml, $50 \mathrm{ng} / \mathrm{ml}$, and $100 \mathrm{ng} / \mathrm{ml}) \mathrm{in}$ -

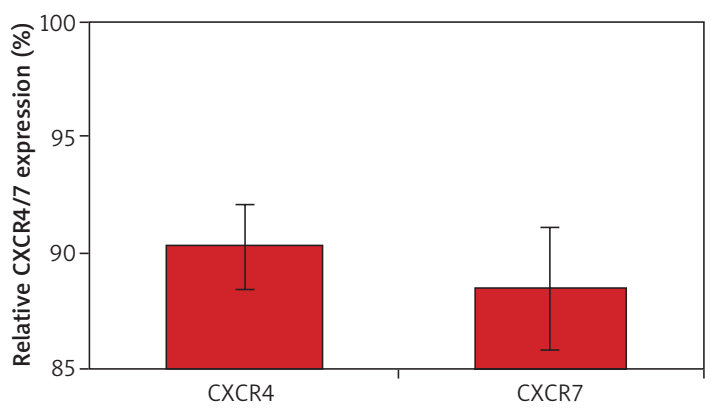

C

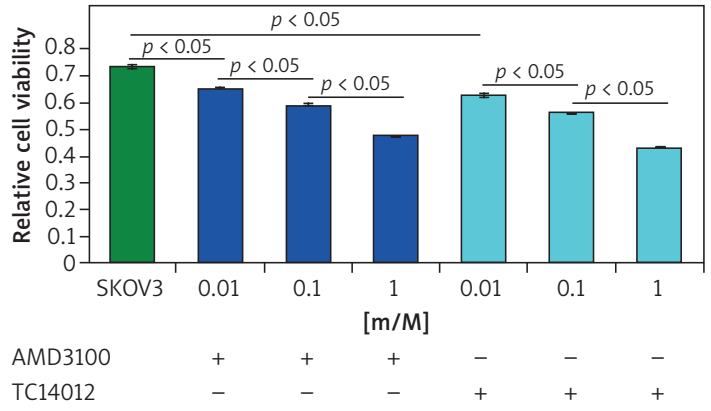

Figure 2. Evaluation for the effects of CXCL12 and CXCR4/CXCR7 inhibitor on cell viability of SKOV3 cells. A - Endogenous expression of CXCR4 and CXCR7 in SKOV3 cells using western blotting assay. B - Effects of CXCL12 on the cell viability of SKOV3 cells at $24 \mathrm{~h}, 48 \mathrm{~h}$, and $72 \mathrm{~h}$. C - Effects of CXCR4 inhibitor (AMD3100) and CXCR7 inhibitor (TC14012) on the cell viability of SKOV 3 cells. ${ }^{*} p<0.05$ vs. the other concentration of CXCL12 
A

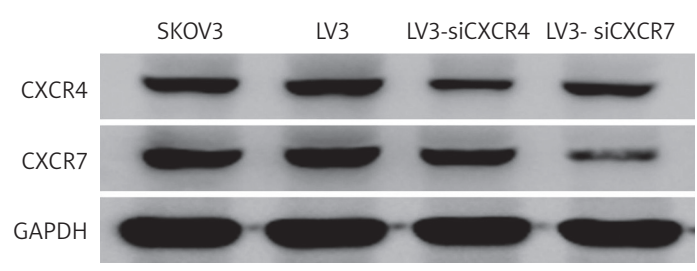

B

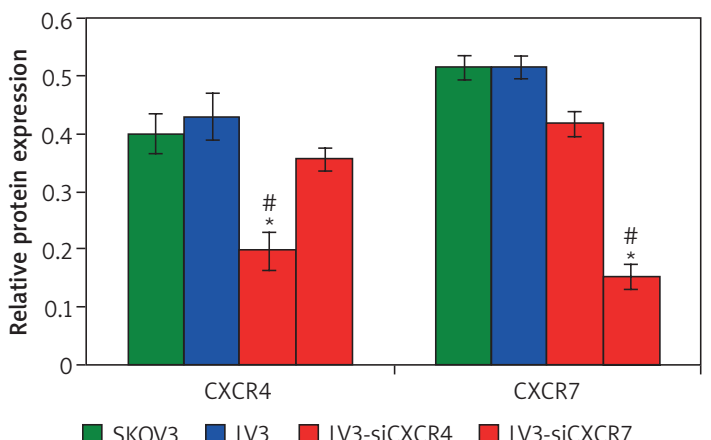

Figure 3. Effects of CXCR4/CXCR7 gene silencing on the CXCR4/CXCR7 protein expression. A - Western blot assay images. B - Statistical analysis for CXCR4 and CXCR7 protein expression. ${ }^{*} p<0.05$ vs. LV3 group, ${ }^{*} p<0.05$ vs. SKOV3 group

A

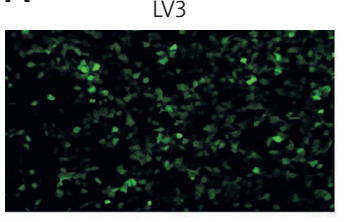

LV3-SiCXCR7

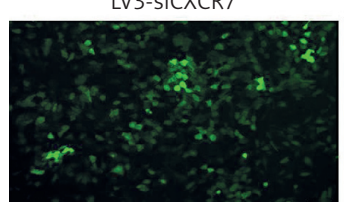

C
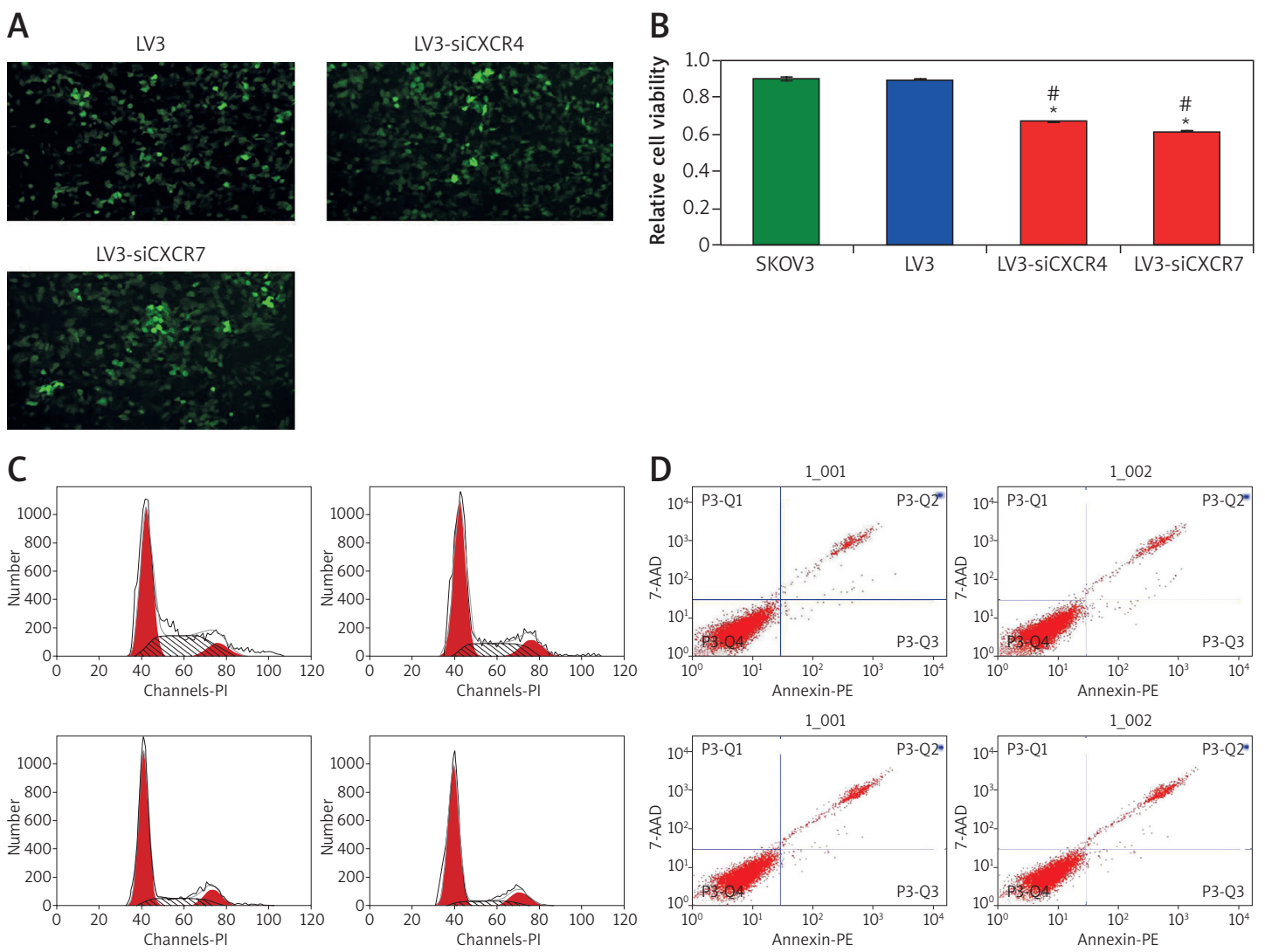
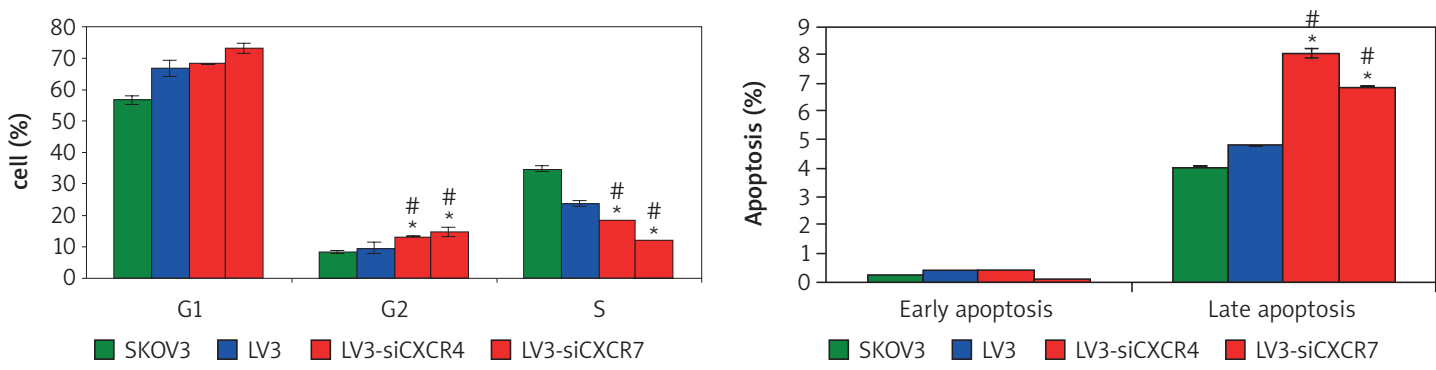

Figure 4. Evaluation for the effects of siCXCR4/siCXCR7 on SKOV3 cell viability, cell cycle, and apoptosis. A - Identification for transfection efficacy of siCXCR4/siCXCR7 plasmids. B - Statistical analysis for cell viability of SKOV3 cells undergoing siCXCR4/siCXCR7 treatment. C - Cell cycle analysis for SKOV3 cells undergoing siCXCR4/siCXCR7 treatment. D - Apoptosis analysis for SKOV3 cells undergoing siCXCR4/siCXCR7 treatment using flow cytometry assay. ${ }^{*} p<0.05$ vs. LV3 group, ${ }^{*} p<0.05$ vs. SKOV3 group 
creased the cell viability of SKOV3 cells; however, only the $100 \mathrm{ng} / \mathrm{ml} \mathrm{CXCL12} \mathrm{treatment} \mathrm{significantly}$ enhanced cell viability of SKOV3 compared to that at other concentrations (Figure $2 \mathrm{~B}, p<0.05$ ). Therefore, in the following experiments, we employed $100 \mathrm{ng} / \mathrm{ml} \mathrm{CXCL12}$ as the optimal concentration for treating SKOV3 cells.

Undergoing the treatment of $100 \mathrm{ng} / \mathrm{ml} \mathrm{CXCL12,}$ both CXCR4 inhibitor (AMD3100) and CXCR7 inhibitor (TC14012) significantly reduced the cell viability of SKOV3 cells compared to that of single SKOV3 cells (Figure $2 \mathrm{C}, p<0.05$ ).

\section{CXCR4/CXCR7 gene silencing decreased CXCR4/CXCR7 protein expression}

The western blot findings identified that the expression of CXCR4 was significantly decreased in the LV3-siCXCR4 group compared to that in the SKOV 3 group and the LV3 group (Figures $3 \mathrm{~A}, \mathrm{~B}$; $p<0.05)$. Meanwhile, expression of CXCR7 was also markedly reduced in the LV3-siCXCR7 group compared to that in the SKOV3 group and the LV3 group (Figures $3 \mathrm{~A}, \mathrm{~B} ; p<0.05$ ). Therefore, both LV3-siCXCR4 and LV3-siCXCR7 were transfected into SKOV3 cells to measure parameters in subsequent experiments.

CXCR4/CXCR7 gene silencing decreased cell viability of SKOV3 cells

The CXCR4/CXCR7 gene silencing plasmids LV3siCXCR4 and LV3-siCXCR7 were transfected into CXCL12-treated SKOV3 cells, and demonstrated higher transfect efficacy (Figure 4 A). Moreover, the LV3-siCXCR4 and LV3-siCXCR7 transfected SKOV3 cells failed to demonstrate or showed very low CXCR4 and CXC7 expression (data now

A

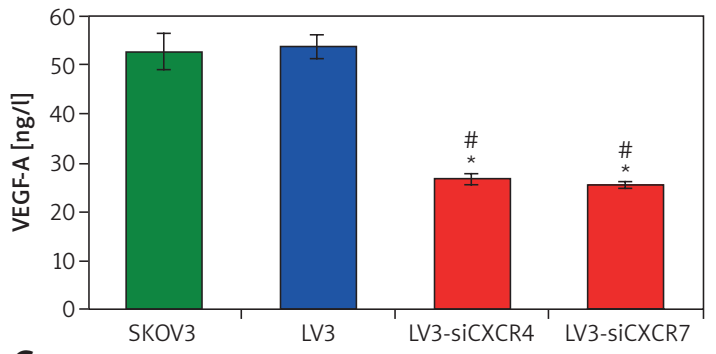

C

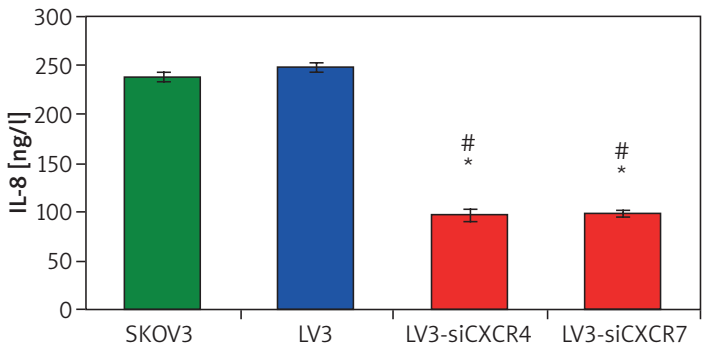

shown). The MTT data showed that the cell viabilities of the LV3-siCXC4 and LV3-siCXCR7 groups were significantly lower than those in the SKOV3 group and LV3 group (Figure $4 \mathrm{~B} ; p<0.05$ ).

\section{CXCR4/CXCR7 gene silencing modulated}

cell cycle and increased cell apoptosis rates

The cell cycle assay results indicated that the percentage of G2 phage cells was significantly increased and the percentage of $S$ phage cells was significantly decreased in the LV3-siCXCR4 and LV3-siCXCR7 groups compared to that of the SKOV3 group and LV3 group (Figure 4 C, $p<0.05$ ). Meanwhile, the flow cytometry assay findings showed that the late apoptosis rates were significantly increased in the LV3-SiCXCR4 and LV3-siCXCR7 groups compared to that in the SKOV3 group and LV3 group (Figure $4 \mathrm{D} ; p<0.05$ ).

\section{CXCR4/CXCR7 gene silencing inhibited VEGF-1, IL-6, and IL-8 secretion}

In order to identify the tumourigenesis characteristics of CXCR4/CXCR7, the cytokines, including VEGF-1, IL-6, and IL-8, were examined in SKOV3 cells. The results showed that VEGF-A (Figure $5 \mathrm{~A}$ ), IL-6 (Figure $5 \mathrm{~B}$ ), and IL-8 (Figure $5 \mathrm{C}$ ) levels in the CXCR4/CXCR7 gene silencing group (LV3-siCXCR4/ LV3-siCXCR7 group) were significantly inhibited compared to those in the SKOV3 group and LV3 group $(p<0.05)$.

\section{CXCR4/CXCR7 gene silencing modulated} the tumourigenesis-associated molecules

In this study, we evaluated the expression of the tumour angiogenesis-associated molecule,

B

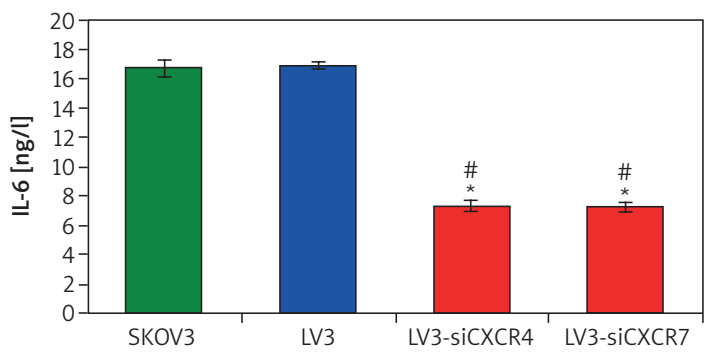

Figure 5. Inflammatory factors, including VEGF-A, IL-6, and IL-8 in SKOV3 cells were detected using ELISA assay. A - Statistical analysis for VEGF-A levels. B - Statistical analysis for IL-6 levels. C - Statistical analysis for IL-8 levels. ${ }^{*} p<0.05$ vs. LV3 group, ${ }^{\#} p<0.05$ vs. SKOV3 group 
CD31, invasion-associated molecules, MMP-9, epithelial-mesenchymal transition (EMT)-associated molecule, E-cadherin and vimentin [21], in CXCR4/ CXCR7 gene-silenced SKOV3 cells. According to the qRT-PCR assay results (Figure 6 A), the CD31, MMP-9, and vimentin expressions were significantly down-regulated, and E-cadherin expression was significantly up-regulated in the LV3-siCXCR4

A
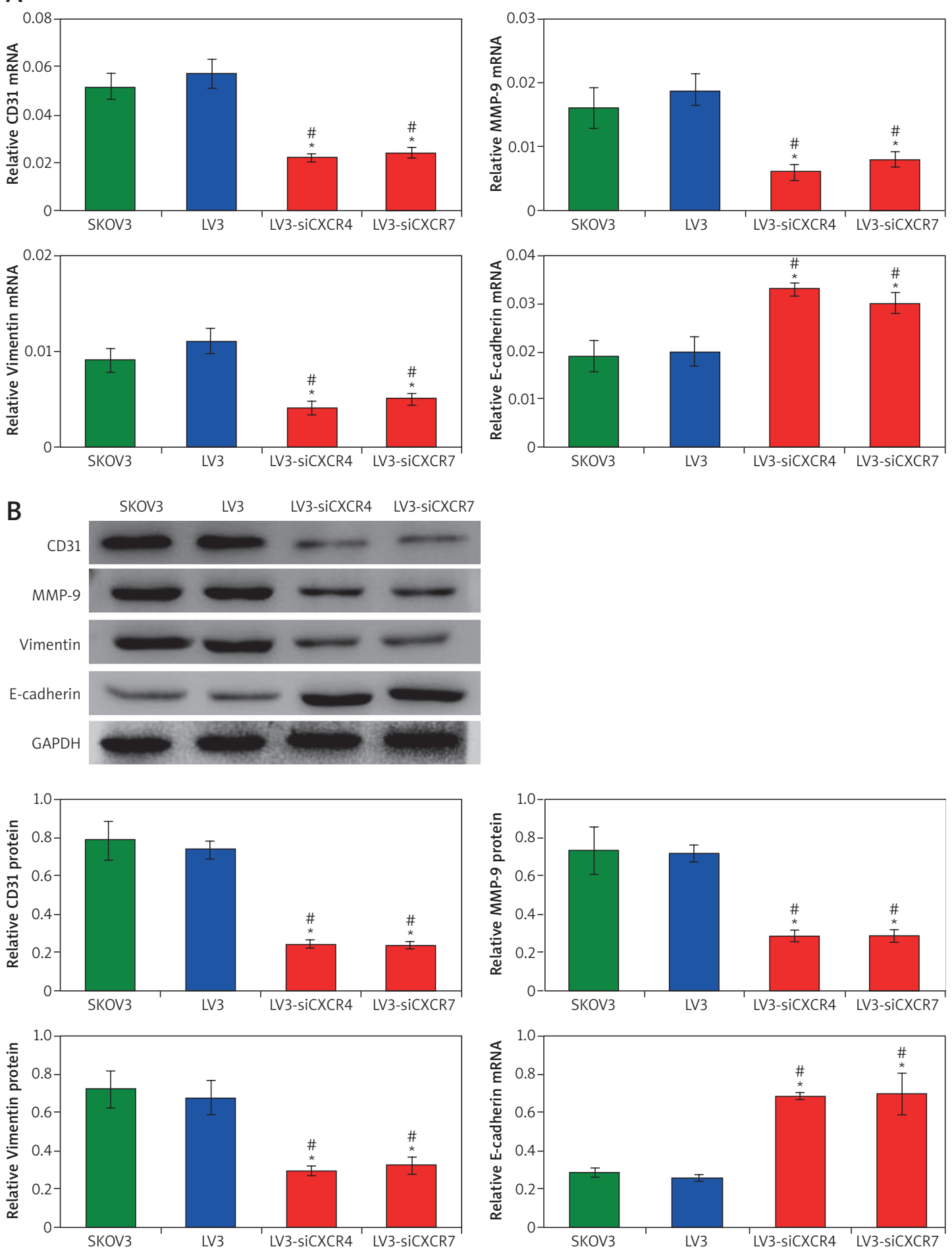

Figure 6. Observation for tumour angiogenesis-associated molecule, CD31, invasion-associated molecules, MMP-9 and EMT-associated molecule, E-cadherin and vimentin in the SKOV3 cells undergoing iCXCR4/siCXCR7 treatment. A - CD31, MMP-9, vimentin, and E-cadherin mRNA expression detected using qRT-PCR assay. B - CD31, MMP-9, vimentin, and E-cadherin protein expression detected using western blotting assay. ${ }^{*} p<0.05$ Vs. LV3 group, ${ }^{\#} p<0.05$ vs. SKOV3 group 
and LV3-siCXCR7 groups compared to that in the SKOV3 group and LV3 group $(p<0.05)$. Meanwhile, the western blot assay findings also indicated the same tendency of CD31, MMP-9, vimentin, and E-cadherin expression in CXCR4/CXCR7 gene-silenced SKOV3 cells (Figure 6 B).

\section{CXCR4/CXCR7 gene silencing suppressed p-ERK1/2 expression}

The tumour cell proliferation and differentiation-associated molecule ERK1/2 and its phosphorylated form (p-ERK1/2) [22] were also examined in the CXCR4/CXCR7 gene-silenced SKOV3 cells using western blot assay (Figure $7 \mathrm{~A}$ ). The results indicated that there were no significant differences for ERK1/2 mRNA transcription among all groups and time points (Figure $7 \mathrm{~B} ; p>0.05$ ). However, the $p$-ERK1/2 mRNA transcriptions were significantly reduced in the LV3-siCXCR4 and LV3siCXCR7 groups compared to those in the SKOV3 group and LV3 group (Figure $7 \mathrm{C} ; p<0.05$ ).

\section{CXCR4/CXCR7 gene silencing strengthened the inhibitive effects of CXCR4/CXCR7 inhibitor on $\mathrm{p}$-ERK1/2 expression}

In order to confirm the effects of CXCR4/ CXCR7 gene silencing on the p-ERK1/2 expression, a western blot assay was conducted (Figure $8 \mathrm{~A}$ ). The results indicated that both CXCR4/ CXCR7 gene inhibitors (AMD3100/TC14012) and CXCR4/CXCR7 siRNAs significantly decreased the p-ERK1/2 expression compared to single CXCL12treated SKOV3 cells (Figure $8 \mathrm{~B} ; p<0.05$ ). The inhibitive effects of CXCR4/CXCR7 gene silencing on $p$-ERK1/2 expression were slightly lower

A

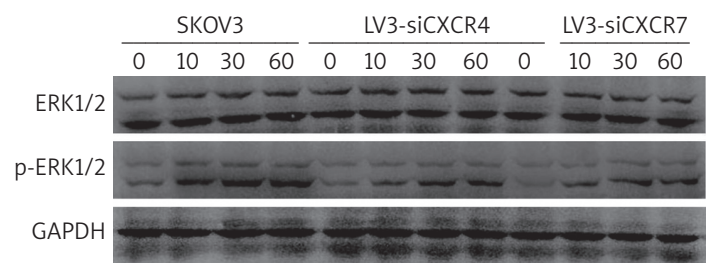

C

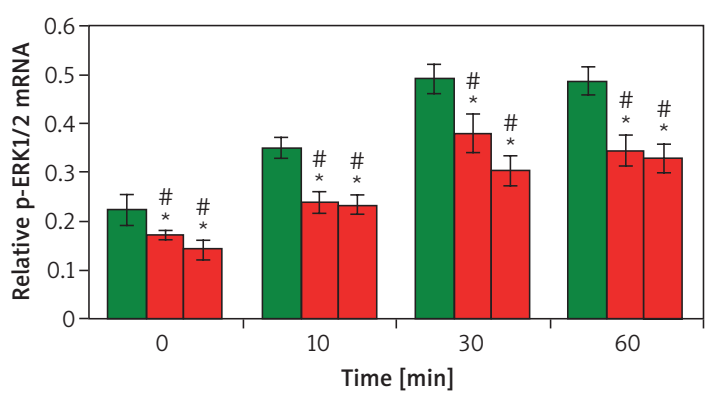

SKOV3 than that in CXCR4/CXCR7 inhibitors (Figure $8 \mathrm{C}$; $p<0.05)$. However, the CXCR4/CXCR7 gene silencing significantly strengthened the inhibitive effects of CXCR4/CXCR7 inhibitor on p-ERK1/2 expression in CXCL12-treated SKOV3 cells (Figure $8 C ; p<0.05)$.

CXCR4/CXCR7 gene silencing reduced tumour volume in the mouse Xenograft model

After the SKOV3 cell transplantation, the tumour growth in the mouse Xenograft model was observed for 26 days (Figure 9 A). The tumour volume in the mouse LV3-siCXCR4/LV3-siCXCR7 group was significantly reduced compared to that in the SKOV3 group and LV3 group, from 12 days to 26 days post transplantation (Figure $9 \mathrm{~B} ; p<0.05$ ). Moreover, the tumour inhibitive rates in the LV3SiCXCR4/LV3-siCXCR7 group were significantly higher compared to the SKOV3 group and LV3 group (Figure $9 \mathrm{C} ; p<0.05$ ).

\section{CXCR4/CXCR7 siRNA transplantation} regulated tumourigenesis-associated molecules in the mouse Xenograft model

In the cancer tissues of the mouse Xenograft model, CD31, MMP-9, E-cadherin, vimentin, and VEGF-A, were examined using immunohistochemistry assay (Figure $10 \mathrm{~A}$ ). The results showed that CXCR4/CXCR7 siRNA transplantation significantly reduced CD31, MMP-9, vimentin, and VEGF-A expression and significantly enhanced E-cadherin expression compared to the single SKOV3 cells and LV3 transplanted mouse Xenograft model (Figure $10 \mathrm{~B} ; p<0.05$ ).

\section{B}

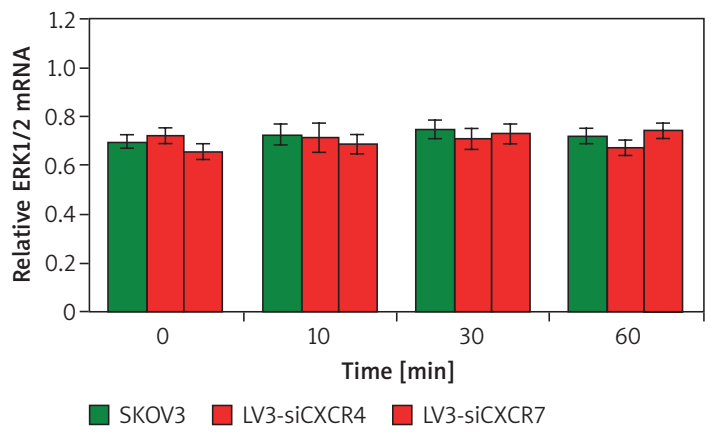

Figure 7. Evaluation for the ERK $1 / 2$ and $p$-ERK $1 / 2$ expression in SKOV3 cells using western blotting assay. A - Western blotting images. B - Statistical analysis for ERK1/2 expression in SKOV3, LV3siCXCR4, and LV3-siCXCR7 groups at different time points. C - Statistical analysis for p-ERK1/2 expression in SKOV3, LV3-siCXCR4, and LV3-siCXCR7 groups at different time points. ${ }^{*} p<0.05$ vs. LV3 group, ${ }^{\#} p<0.05$ vs. SKOV3 group 
A

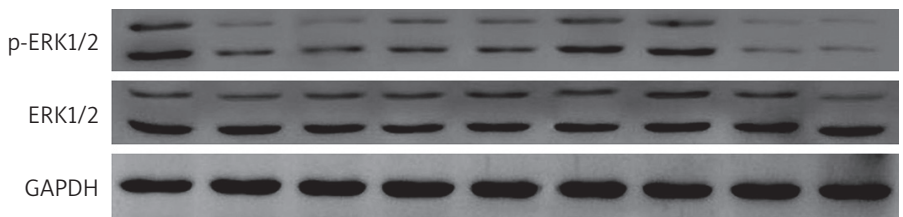

B

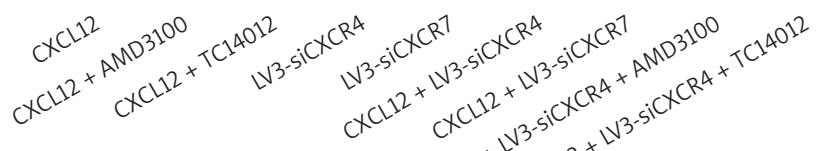
$\mathrm{CxCl} 2+\mathrm{CxCl}^{2}$
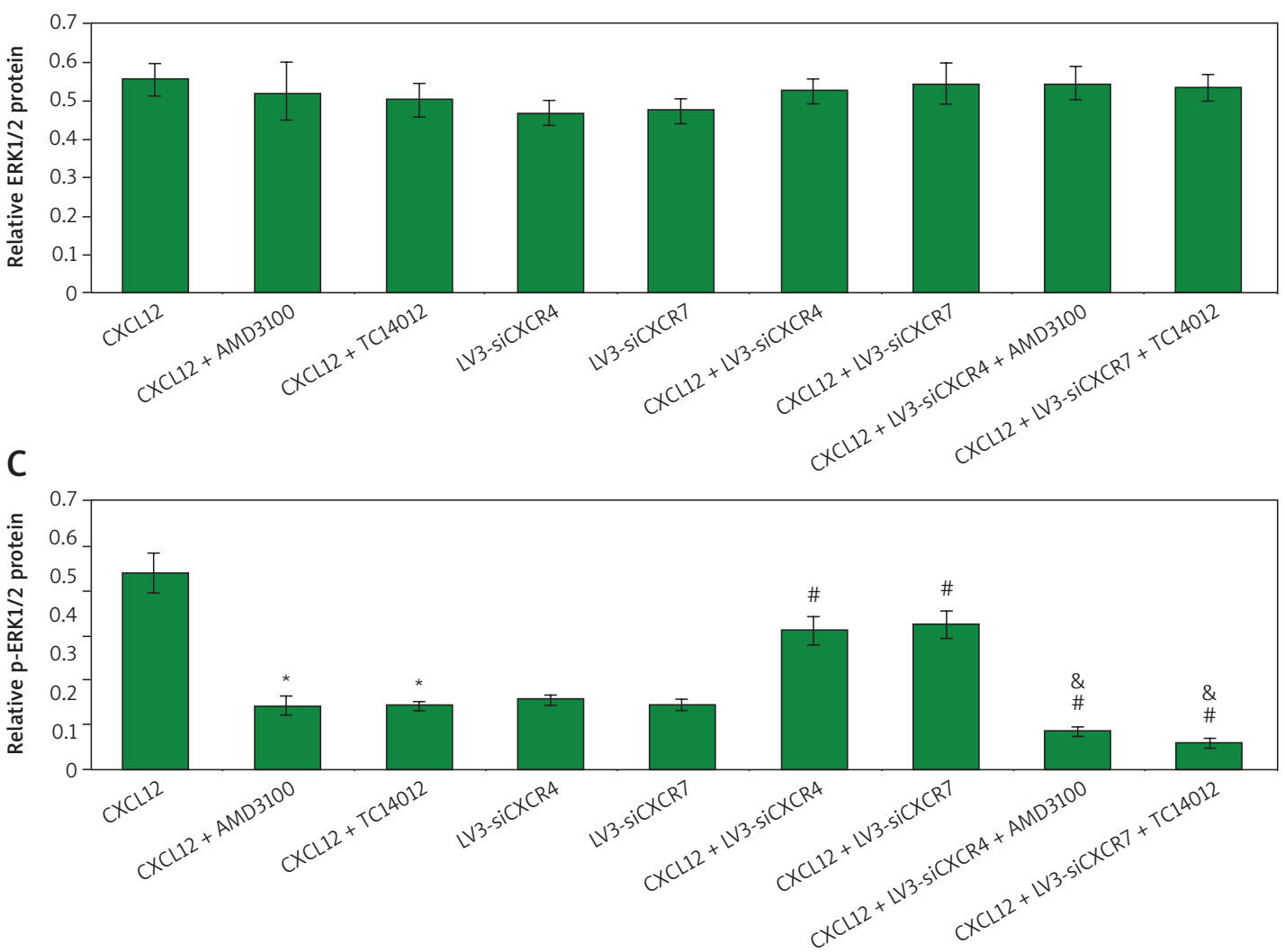

Figure 8. Determination for the ERK1/2 and p-ERK1/2 expression in SKOV3 cells undergoing CXCL12 and/or siCXCR4/siCXCR7 treatment (or AMD3100 or TC14012) using western blotting assay. A - Western blot images. B - Statistical analysis for ERK1/2 expression in various groups. C - Statistical analysis for $\mathrm{p}$-ERK1/2 expression in various groups. ${ }^{*} p<0.05$ vs. CXCL12 group, ${ }^{*} p<0.05$ vs. CXCL12 + AMD3100/TC14012 group; $p<0.05$ vs. CXCL12 + LV3siCXCR4/LV3-siCXCR7 group

A

SKOV3

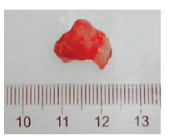

LV3

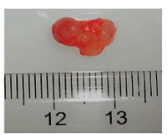

C

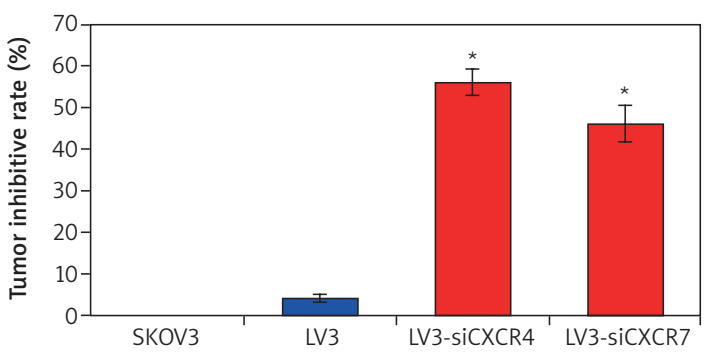

B

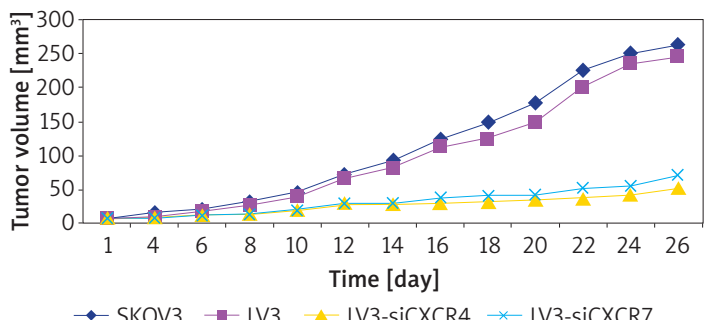

Figure 9. Tumour volume assessment for the mouse Xenograft model from day 1 to day 26. A - Isolated tumours of mouse Xenograft model. B - Statistical analysis for the tumour volume of mouse Xenograft model. C - Statistical analysis for tumour inhibitive rates. ${ }^{*} p<0.05$ vs. LV3 group 
A
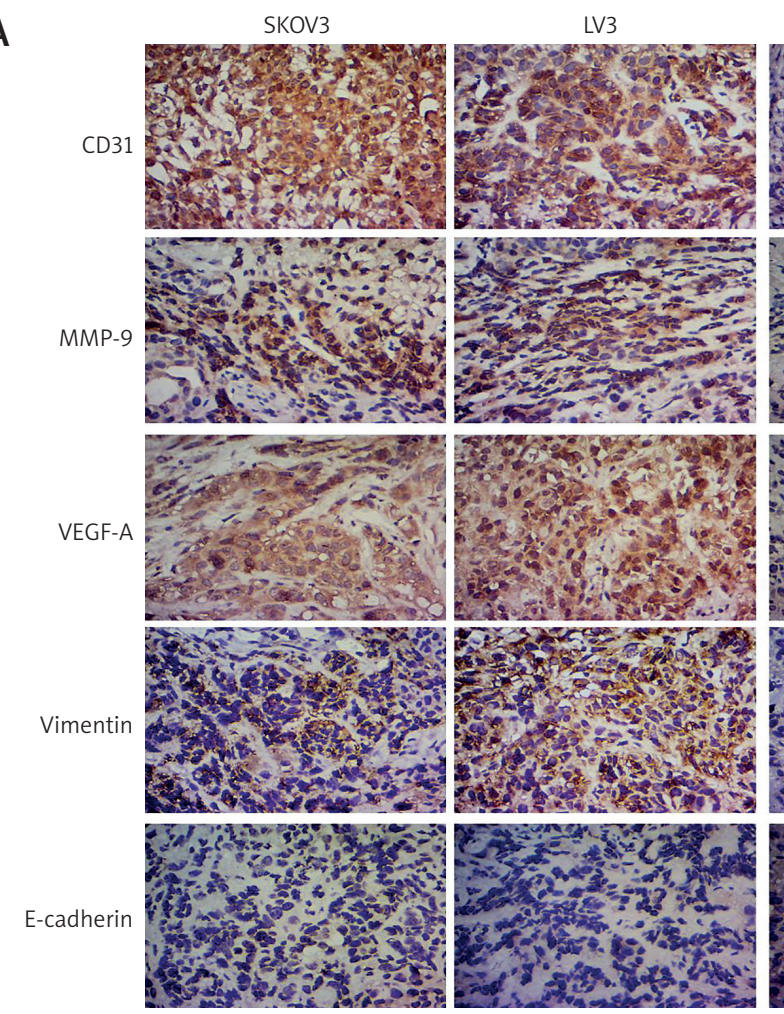

B

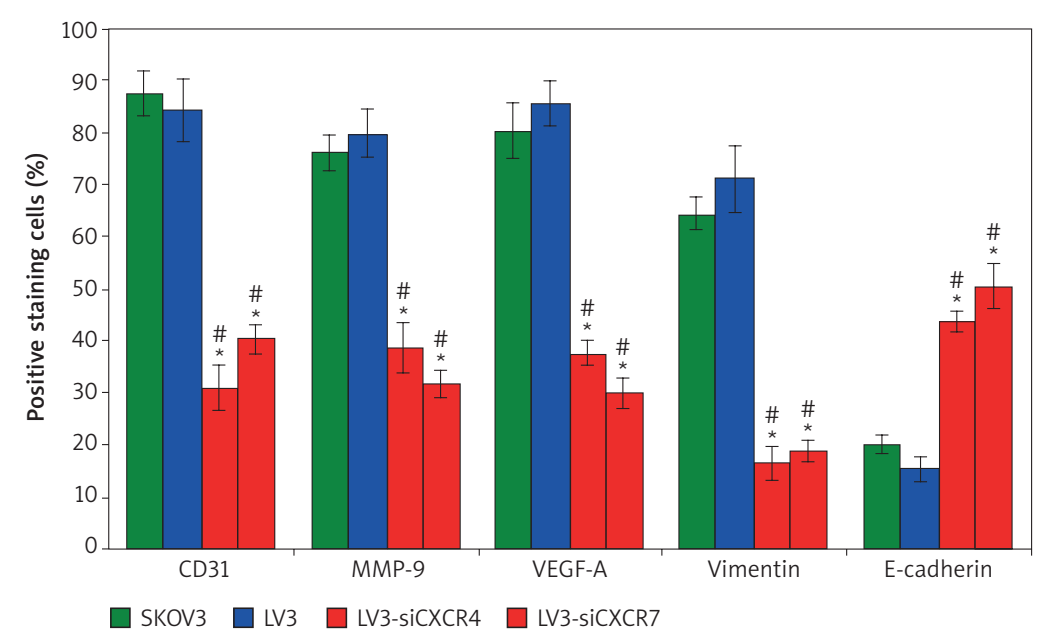

Figure 10. Immunohistochemistry assay for detecting CD31, MMP-9, VEGF-A, vimentin, and E-cadherin expression in the tumour tissues of mouse Xenograft model. A - Immunohistochemistry assay staining images. B - Statistical analysis for the positive staining cells. ${ }^{*} p<0.05$ vs. LV3 group, ${ }^{*} p<0.05$ vs. SKOV3 group

\section{Discussion}

Ovarian cancer is considered to be the most common malignancy and the most deadly gynaecological among females [23]. Although the clinical surgery might be curative for early-stage cancers, the therapeutic strategies for treating late-stage cancers are limited by lower efficacy [24]. The present study synthesised the CXCR4/CXCR7-silencing siRNAs and investigated their possible therapeutic values against ovarian cancer and associated signalling pathways, both in vivo and in vitro levels.
According to the reported correlation between the CXCL12-CXCR4/CXCR7 chemokine axis and cancer development or progression [7, 25], the effects of CXCL12, CXCR4, and CXCR7 on cell viability of SKOV3 cells were determined in this study. CXCL12 administration resulted in a dosedependent increase for the SKOV3 cell viability. Meanwhile, the CXCR4 inhibitor (AMD3100)- and CXCR7 inhibitor (TC14012)-treated SKOV3 cells also demonstrated dose-dependent enhancement of cell viabilities. These results suggest that the CXCL12 treatment and CXCR4/CXCR7 gene silenc- 
ing are associated with the tumour cell proliferation of SKOV3 cells. For the CXCR12 treatment, the concentration of $100 \mathrm{ng} / \mathrm{ml}$ has been proven to be the optimal dosage for enhancing the cell proliferation of SKOV3 cells, which was used to treat SKOV3 cells in the following experiments.

In order to mimic the effects of CXCR4/CXCR7 inhibitor, the small interfering RNAs against CXCR4 and CXCR7 gene (SiCXCR4 and SiCXCR7) were synthesised and transfected into SKOV3 cells. The results illustrated that both SiCXCR4 and SiCXCR7 treatment decreased cell viability of SKOV3 cells, the effects of which are consistent with CXCR4/ CXCR7 inhibitors. The cell cycle was initiated when the SKOV3 cells were administrated with siCXCR4 and SiCXCR7 (blocking the arrested cell cycle of SKOV3 cells), illustrating the decreased $\mathrm{S}$ phage cells and increased $G 2$ phage cells, which is consistent with the effects of CXCR4/CXCR7 inhibitors previously reported [26]. Moreover, a remarkable increase for the apoptosis, especially for the late apoptosis, was discovered in the CXCL12-treated SKOV3 cells following SiCXCR4/SiCXCR7 administration. These findings suggest that the siCXCR4/ siCXCR7 administration potently inhibited the tumour cell proliferation by inducing the SKOV3 cell apoptosis. Also, the tumourigenesis associated pro-inflammatory factors, including VEGF-A, IL-6, and IL-8 [27], were evaluated in the SiCXCR4/siCXCR7-treated SKOV3 cells. Our results showed that CXCR4/CXCR7 gene silencing obviously inhibited the VEGF-1, IL-6, and IL-8 secretion of the CXCL12treated SKOV3 cells, which suggests that the siCXCR4/siCXCR7 administration also demonstrated anti-tumour effects by alleviating the inflammatory response of ovarian cancer cells. In fact, blocking the pro-inflammatory or inflammatory signalling pathways in cancer cells is a critical approach for suppressing tumour cell proliferation [28].

Furthermore, the tumour angiogenesis-associated molecule, CD31, and tumour distant metastasis-related molecule [21, 29], were also detected in siCXCR4/siCXCR7-administrated SKOV3 cells. We found that CD31 and MMP-9 production was significantly inhibited in the SKOV3 cells undergoing siCXCR4/siCXCR7 administration, which suggests that the SiCXCR4/siCXCR7 blocks the tumour angiogenesis by suppressing CD31 and MMP-9 expression. A previous study [7] reported that the CXCR4 and CXCR7 play key roles in the CXCR12-activated EMT phenotypes. The present study also showed that the EMT-associated molecule, vimentin, was significantly decreased, and E-cadherin was significantly increased in siCXCR4/siCXCR7-treated SKOV3 cells. These results hint that the CXCL12CXCR4 and CXCL12-CXCR7 chemokine axis represents a potential therapeutic strategy to inhibit development and progression of ovarian cancer.
The mitogen-activated protein kinase (MAPK) signalling pathway could modulate a number of processes of tumour growth and metastasis [30], especially for the ERK1/2 molecule, which has been proven to play a critical role in cancer metastasis [31]. In this study, phosphorylation of ERK1/2 was activated and decreased by the siCXCR4/siCXCR7 administration in CXCR12-activated SKOV3 cells. Moreover, our results also illustrated that the CXCR4/CXCR7 gene silencing strengthened the inhibitive effects of CXCR4/CXCR7 in hibitor (AMD3100 and TC14012) on reduction of $p$ ERK1/2 expression. Our findings are in accordance with the participation of MAPK in cancer development and progression [32]. Although the specific mechanism for the function of CXCR4/CXCR7 in signal transduction has not been fully clarified in this study, we speculate that CXCR4/CXCR7 might act as an intermediate molecule by activating the ERK1/2 directly or indirectly. In summary, our findings suggest that siCXCR4/siCXCR7 might regulate the MAPK signalling pathway in ovarian cancer.

Moreover, the tumour cell SKOV3 cells transplanted mouse Xenograft model was also established in this study. This study observed that siCXCR4/siCXCR7 administration significantly reduced the tumour volume and demonstrated higher tumour inhibitive rates of the SKOV3 cells transplanted mouse Xenograft model. Meanwhile, the tumour angiogenesis, metastasis, and EMTcorrelated molecules [7, 21, 29], including CD31, MMP-9, VEGF-A, and vimentin, were significantly increased, and E-cadherin was significantly decreased in the tumour tissues of the mouse Xenograft model. However, we did not employ more specific methods (such as western blot assay) to detect CD31, MMP-9, VEGF-A, vimentin, and E-cadherin expressions in tumour tissues, which is a limitation of our study. These results suggest that siCXCR4/siCXCR7 administration could inhibit tumour angiogenesis, tumourigenesis, and progression of ovarian cancer, and afre partially consistent with other studies focusing on other cancers, including lung cancer [33], breast cancer [34], and hepatocellular carcinoma [35]. Moreover, the sample size for the mouse Xenograft models was relatively small in each group, which is also a limitation of our study. In a subsequent study, we would enlarge the sample size of the mouse Xenograft models.

In conclusion, this study identified siCXCR4/ CXCR7 as a potential therapeutic molecule to suppress ovarian cancer cell proliferation in vitro and tumour growth of a mouse Xenograft model. The anti-tumour effects of siCXCR4/CXCR7 are mainly mediated by CXCL12-CXCR4/CXCR7 chemokine axis and MAPK signalling pathway. The present data highlight the promising application of CXCR4/ 
CXCR7 gene silencing as a potential therapeutic approach for inhibiting ovarian cancer growth.

\section{Conflict of interest}

The authors declare no conflict of interest.

\section{References}

1. Torre LA, Trabert B, DeSantis CE, et al. Ovarian cancer statistics, 2018. CA Cancer J Clin 2018; 68: 284-96.

2. Zhihong Z, Ruibin C, Liping L, et al. MicroRNA-1179 regulates proliferation and chemosensitivity of human ovarian cancer cells by targeting the PTEN-mediated PI3K/ AKT signaling pathway. Arch Med Sci 2019; 16: 907-14.

3. Buranjiang G, Kuerban R, Abuduwanke, et al. MicroRNA331-3p inhibits proliferation and metastasis of ovarian cancer by targeting RCC2. Arch Med Sci 2019; 15: 1520-9.

4. Ledermann JA, Raja FA, Fotopoulou C, et al. Newly diagnosed and relapsed epithelial ovarian carcinoma: ESMO clinical practice guidelines for diagnosis, treatment and follow-up. Ann Oncol 2013; 24 Suppl 6: vi24-32.

5. Chien J, Poole EM. Ovarian cancer prevention, screening, and early detection: report from the 11th Biennial Ovarian Cancer Research Symposium. Int J Gynecol Cancer 2017; 27: S20-2.

6. Chen $\mathrm{Y}, \mathrm{Du} \mathrm{H}$. The promising PARP inhibitors in ovarian cancer therapy: from olaparib to others. Biomed Pharmacother 2018; 99: 552-60.

7. Zheng N, Liu W, Chen J, et al. CXCR7 is not obligatory for CXCL12-CXCR4-induced epithelial-mesenchymal transition in human ovarian cancer. Mol Carcinog 2019; 58 144-55.

8. Werner TA, Forster CM, Dizdar L, et al. CXCR4/CXCR7/ CXCL12 axis promotes an invasive phenotype in medullary thyroid carcinoma. Br J Cancer 2017; 117: 1837-45.

9. Grundker C, Bauerschmitz G, Knapp J, et al. Inhibition of SDF-1/CXCR4-induced epithelial-mesenchymal transition by kisspeptin-10. Breast Cancer Res Treat 2015; 152: 41-50.

10. Wang J, Shiozawa Y, Wang J, et al. The role of CXCR7/ RDC1 as a chemokine receptor for CXCL12/SDF-1 in prostate cancer. J Biol Chem 2008; 283: 4283-94.

11. Hattermann K, Held-Feindt J, Lucius R, et al. The chemokine receptor CXCR7 is highly expressed in human glioma cells and mediates antiapoptotic effects. Can Res 2010; 70: 3299-308.

12. Miao Z, Luker KE, Summers BC, et al. CXCR7 (RD C1) promotes breast and lung tumor growth in vivo and is expressed on tumor-associated vasculature. Proc Natl Acad Sci USA 2007; 104: 15735-40.

13. Hattermann K, Mentlein R. An infernal trio: the chemokine CXCL12 and its receptor CXCR4 and CXCR7 in tumor biology. Ann Anat 2013; 195: 103-10.

14. Aiuti A, Webb IJ, Bleul C, et al. The chemokine SDF-1 is a chemoattractant for human CD34+ hematopoietic progenitor cells and provides a new mechanism to explain the mobilization of CD34+ progenitors to peripheral blood. J Exp Med 1997; 185: 111-20.

15. Kryczek I, Wei S, Keller E, et al. Stroma-derived factor (SDF-1/CXCL12) and human tumor pathogenesis. Am J Physiol Cell Physiol 2007; 292: C987-95.

16. Yin Q, Jin P, Liu X, et al. SDF-1 alpha inhibits hypoxia and serum deprivation-induced apoptosis in mesenchymal stem cells through PI3K/Akt and ERK1/2 signaling pathways. Mol Biol Rep 2011: 38: 9-16.
17. Zhang L, Wang Y, Ma J, et al. Exogenous MSCs ameliorate hypoxia/reoxygenation injury in renal tubular epithelial cells through JAK/STAT signaling pathway-mediated regulation of HMGB1. Am J Transl Res 2017; 9: 2412-20.

18. Li S, Dong P, Wang J, et al. Icariin, a natural flavonol glycoside, induces apoptosis in human hepatoma SMMC7721 cells via a ROS/JNK-dependent mitochondrial pathway. Cancer Lett 2010; 298: 222-30.

19. Bao R, Shu Y, Wu X, et al. Oridonin induces apoptosis and cell cycle arrest of gallbladder cancer cells via the mitochondrial pathway. BMC Cancer 2014; 14: 217.

20. Livak KJ, Schmittgen TD. Analysis of relative gene expression data using real-time quantitative PCR and the 2- $\Delta \Delta$ ct method. Methods 2001; 25: 402-8.

21. Gao Y, Zhao XL, Gu Q, et al. Correlation of vasculogenic mimicy with clinicopathologic features and prognosis of ovarian carcinoma. Zhonghua Bing Li Xue Za Zhi 2009; 38: 585-9.

22. Xu H, Zhao H, Yu J. HOXB5 promotes retinoblastoma cell migration and invasion via ERK1/2 pathway-mediated MMPs production. Am J Transl Res 2018; 10: 1703-12.

23. Oberaigner W, Minicozzi P, Bielska-Lasota M, et al. Survival for ovarian cancer in Europe: the across-country variation did not shrink in the past decade. Acta Oncol 2012; 51: 441-53.

24. Hu MI, Ying AK, Jimenez C. Update on medullary thyroid cancer. Endocrinol Metab Clin North Am 2014; 43: 423-42.

25. Yuan C, Liu X, Li R, et al. Analysis of the association between the XRCC2 rs3218536 polymorphism and ovarian cancer risk. Arch Med Sci 2020; 16: 682-91.

26. Mao TL, Fan KF, Liu CL. Targeting the CXCR4/CXCL12 axis in treating epithelial ovarian cancer. Gene Ther 2017; 24: 621-9.

27. Khare P, Bose A, Singh P, et al. Gonadotropin and tumorigenesis: direct and indirect effects on inflammatory and immunosuppressive mediators and invasion. Mol Carcinog 2017; 56: 359-70.

28. Zhang Y, Li Y, Li H, et al. Clostridium difficile toxin B recombinant protein inhibits tumor growth and induces apoptosis through inhibiting $\mathrm{BCl}-2$ expression, triggering inflammatory responses and activating C-erbB-2 and Cox-2 expression in breast cancer mouse model. Biomed Pharmacother 2018; 101: 391-8.

29. Zhang LQ, Lv RW, Qu XD, et al. Aloesin suppresses cell growth and metastasis in ovarian cancer SKOV3 cells through the inhibition of the MAPK signaling pathway. Anal Cell Pathol 2017; 2017: 8158254.

30. Wagner EF, Nebreda AR. Signal integration by JNK and MAPK pathways in cancer development. Nat Rev Cancer 2009; 9: 537-49.

31. Islam M, Sharma S, Teknos TN. RhoC regulates cancer stem cells in head and neck squamous cell carcinoma by overexpressing IL-6 and phosphorylation of STAT3. PLoS One 2014; 9: e88527.

32. Islam M, Lin G, Brenner JC, et al. RhoC expression and head and neck cancer metastasis. Mol Cancer Res 2009; 7: 1771-80.

33. Katsura M, Shoji F, Okamoto T, et al. Correlation between CXCR4/CXCR7/CXCL12 chemokine axis expression and prognosis in lymph-node-positive lung cancer patients. Cancer Sci 2018; 109: 154-65.

34. Xu D, Li R, Wu J, et al. Drug design targeting the CXCR4/ CXCR7/CXCL12 pathway. Curr Top Med Chem 2016; 16: 1441-51.

35. Lin L, Han MM, Wang F, et al. CXCR7 stimulates MAPK signaling to regulate hepatocellular carcinoma progression. Cell Death Dis 2014; 5: e1488. 